\title{
EnAPlug - An Environmental Awareness Plug to Test Energy Management Solutions for Households
}

\author{
Luis Gomes $^{1}$, Filipe Sousa ${ }^{1}$, and Zita Vale ${ }^{1}$ \\ ${ }^{1}$ GECAD - Research Group on Intelligent Engineering and Computing for Advanced Innova- \\ tion and Development, Institute of Engineering - Polytechnic of Porto (ISEP/IPP), \\ Rua Dr. António Bernardino de Almeida, 431, 4249-015 Porto, Portugal \\ $\{$ lufog, ffeso, zav\}@isep.ipp.pt
}

\begin{abstract}
The present paper presents a new kind of Smart Plug that covers the needs of power systems R\&D centers. EnAPlug, described in this paper, enables the monitor and control of loads, as a normal Smart Plug. However, it has a great benefit in comparison with a normal Smart Plug, the EnAPlug allows the integration of a variety of sensors so the user can understand the load and the surrounding environment (using a set of sensors that better fit the load). The sensors are installed in the load itself, and must have a clear fit to the load. The paper presents a demonstration of an EnAPlug used in a refrigerator for a demand response event participation, using the sensor capability to measure important values, such as, inside temperature.
\end{abstract}

Keywords: Demand response participation $\cdot$ Multi-agent system $\cdot$ Smart plug

\section{Introduction}

The power system paradigm has been changing in the last years and will continue to change in future years, resulting in the appearance of smart grids [1]. The centralization of generation will end, appearing decentralized generation [2]. The endconsumers will be incentivized to actively participate in smart grids, in a win-win situation, changing completely their roles in today's paradigm [3]. One of the aspects of smart grids is the integration of microgrids.

The proliferation of microgrids started using use cases around the world [4,5], and now this concept can be seen and analyzed in real scenarios. The transaction from scientific concepts towards real implementations, such as [6], is a positive step ena-

The present work has been developed under the EUREKA - ITEA2 Project M2MGrids (ITEA-13011), Project SIMOCE (ANI|P2020 17690), and has received funding from FEDER Funds through COMPETE program and from National Funds through FCT under the project UID/EEA/00760/2013 and SFRH/BD/109248/2015. 
bling the validation of theoretical methodologies in real and uncontrollable environments.

Other important aspect for smart grid successful implementations is the application of Demand Response (DR) programs to give an active role to the small and medium players (usually households or small offices) [7]. The use of DR programs brings clear advantages to smart grids and microgrids [8]. Some use of DR can be found in [9]. Nevertheless, to increase DR dissemination and to promote the appearance of new programs, automatic and intelligent responses must be implemented in the consumer side, specially the small and medium players.

The application of Smart Homes such as in [10] and [11], brings advantages for energy management inside the households, but must important, can increase the DR programs participation using intelligent response methodologies. The development and application of autonomous and intelligent methodologies, for DR users' response, must consider the users impact (particularly the negative impact provoked).

\section{Background of the Proposal}

To implement a Smart Home, monitoring and controlling units are needed, such as, Smart Plugs. At this moment is possible to find a significant number of Smart Plugs available on the market. However, must of them had limitations, such as: not having monitoring; or just monitors the current; or having a closed system without any API.

The use of Smart Plugs in R\&D centers can be done using software like Home Assistant $^{1}$. This is an open source software that aggregates various Internet of Things (IoT) devices into a unique system, while provides a RESTFul interface to monitor and control devices. However, if the R\&D center intention is to study intelligent methodologies in the energy management of the household (for instance, to participate in DR programs), more data and new types of intelligent load control are needed. And for this situation, there is not a suitable Smart Plug in the market.

This paper presents the Environmental Awareness Plug (EnAPlug), a Smart Plug that can be easily developed in R\&D centers fulfilling the center needs and proposes. EnAPlug combines actuators to control the load, and sensors, to monitor not only the load status and energy but also the environment that surrounds the load.

\section{Environmental Awareness Plug}

EnAPlug was idealized and developed for R\&D centers that have a need to test energy management solutions in households. The development of EnAPlug enables R\&D centers to overcome the limitations of the Smart Plugs available on the market, with a costume made solution with context awareness capabilities. EnAPlug can also be used outside R\&D centers for load monitoring and control, enabling a context awareness monitoring of a specific energy resource, for instance, our home kitchen oven.

\footnotetext{
${ }^{1}$ https://home-assistant.io/
} 
The premises of EnAPlug was to build a modular plug that can work with several actuators and sensors while being open for other systems. For this reason, the control and monitor of EnAPlug is open and can be accessible with GET and POST requests.

Fig. 1 shows EnAPlug overall architecture. The light blue block that identifies the microcontroller was implemented using an Arduino Mega 2560 R3, nonetheless other microcontrollers can be used. The microcontroller is the processing unit of EnAPlug and has the following requirements: a serial communication port; at least 1 digital output for the load control; the capability to have TCP/IP connection (using a compatible module); and some digital and analog inputs for sensors (it can also provide communication protocols, such as, I2C).

The yellow blocks are connected to the $230 \mathrm{~V} / \mathrm{AC}$. The Energy Analyzer must be compatible with Modbus/RTU protocol. This requirement will enable the microcontroller to communicate with the energy analyzer using a simple MAX485 component that converts serial communications into RS-485 communications, and vice-versa. The Controller block can be a relay, providing on/off control, or other kind of control, such as, a dimmer. For this paper, it will be used a relay with a 5V/DC coil. The orange block represents the load that we want to monitor and control.

The green and red blocks are external blocks of EnAPlug and are connected using the TCP/IP connection available in EnAPlug. The Control Signal is made using a socket connection to the microcontroller IP on port 80. The Server block is an external server that receives JSON messages and save them in a SQL Server database. The period of storages is defined by EnAPlug.

The sensors blocks are the sensors connected to the Microcontroller that are suitable for the measured load. The idea of the sensors is to give a better knowledge about the measuring Load. For instance, if the intention is to monitor and control a lamp, it is recommended to use a movement sensor and a clarity sensor. The sensors placed must increase the knowledge regarding the load and its context. If we know the load context is possible to perform an intelligent control.

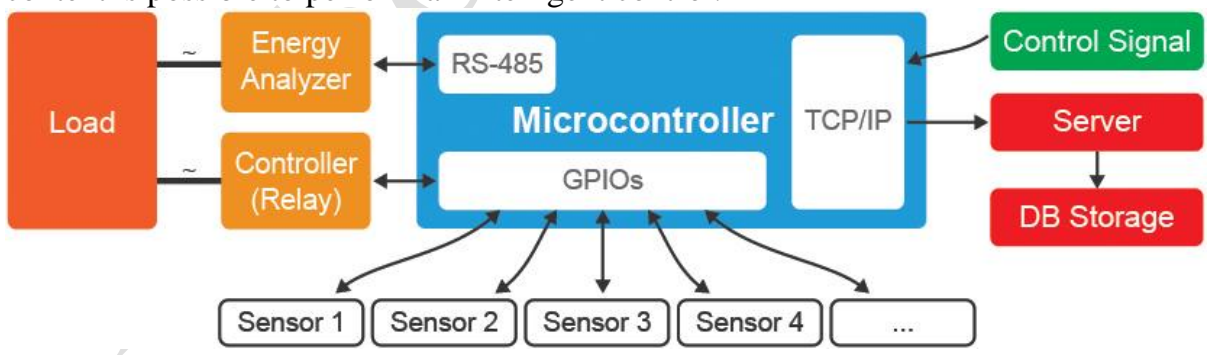

Fig. 1. EnAPlug overall architecture

EnAPlug is a device that understand their environment, enabling an intelligent and a more efficient control. However, the environment awareness capability is only possible with the right sensors. For instance, if the goal is to measure a television, a temperature sensor is not adequate. Therefore, to understand a television more appropriated sensors should be chosen, such as, presence, clarity and noise sensors. 


\section{Demonstration}

For this demonstration, EnAPlug was integrated in the Multi-Agent Smart Grid Platform (MASGriP) [12]. MASGriP has the capability to represent small and medium players in a microgrid scenario. For this scenario MASGriP will be used as a connected microgrid with three players, representing our R\&D buildings (Fig. 2). This representation of MASGriP can be seen in works, such as, $[6,13,14,15]$. In our R\&D center, namely building N, two EnAPlugs were installed and connected to its representative agent:

- EnAPlug for refrigerator (Fig. 3) - for this EnAPlug is used on/off control of the entire refrigerator (including the inside lamp), an energy analyzer to monitor power, reactive power, voltage and current and four sensors: inside temperature and humidity sensor; an outside temperature sensor; and a door opener detector using a clarity sensor;

- EnAPlug for water heater - for this EnAPlug is used a temperature sensor to monitor the water temperature, an on/off controller. The stored energy readings are power, reactive power, voltage and current.

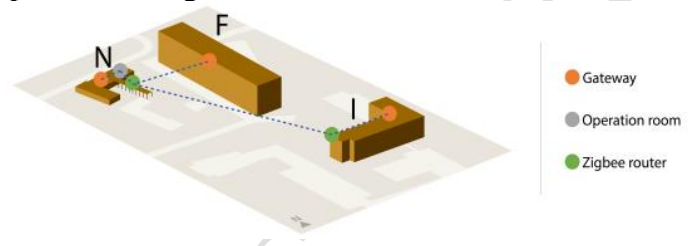

Fig. 2. GECAD MASGriP configuration

EnAPlug readings, from the energy analyzer and from the sensors, are stored in a database each five seconds. A MASGriP agent, that represents building $\mathrm{N}$, uses that data for energy management and gives direct control signals to the EnAPlug.
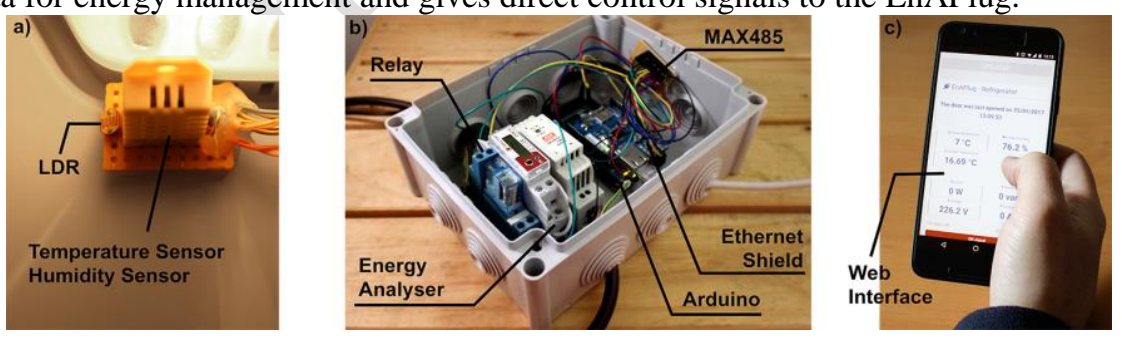

Fig. 3. EnAPlug installed in the refrigerator: a) inside sensors, b) EnAPlug, c) User interface

An external DR event was trigger in MASGriP from 05:00 p.m. to 06:00 p.m.. The event demands to turn off of the refrigerators within this hour. In Fig. 4 is shown the results of the refrigerator connected to EnAPlug, where is visible the DR event and the turn off the refrigerator.

During the day is possible to see, in Fig. 4, the refrigerator light turning on (peaks in blue line of consumptions) when the door is open (red line below). During the event 
EnAPlug detects an open door but there is no increase of consumption, meaning that the light was off. The inside temperature (purple line) was stable and did not increase beyond the refrigerator limit. The DR event was a success as we can see using the consumption line and the inside temperature line. The EnAPlug can detect dangerous situations, such as, the increase of inside temperature and turn the refrigerator back on in an emergency. In this case, the situation was controlled and no damage was made. The results show that after DR event, when the refrigerator was turned on again, the motor started immediately to decrease the inside temperature.

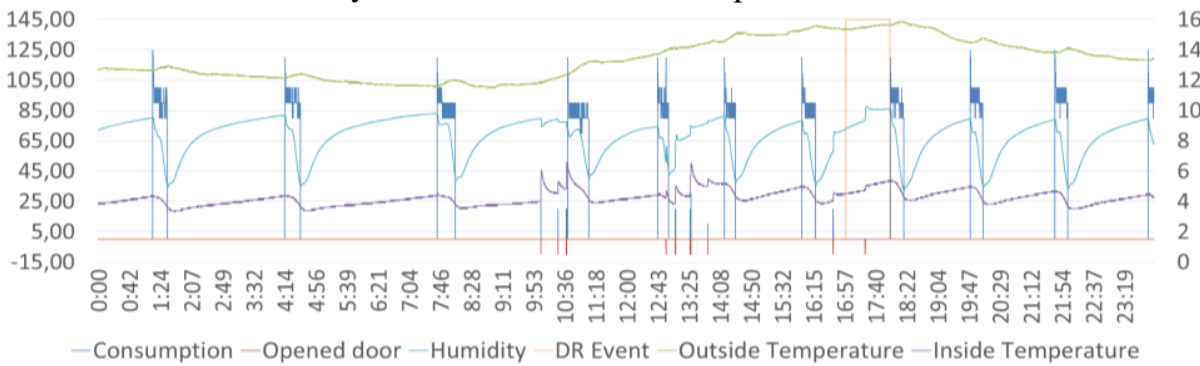

Fig. 4. Refrigerator readings between 00:00 a.m. and 23:59 p.m.

In Fig. 5 is shown the water heater during 24 hours. The temperature sensor is placed outside the water heater (for security reasons), glued in the water pipe. The consumption and the temperature increases when the water heater turned on (06:45). The temperature also increases when a person uses hot water (08:30).

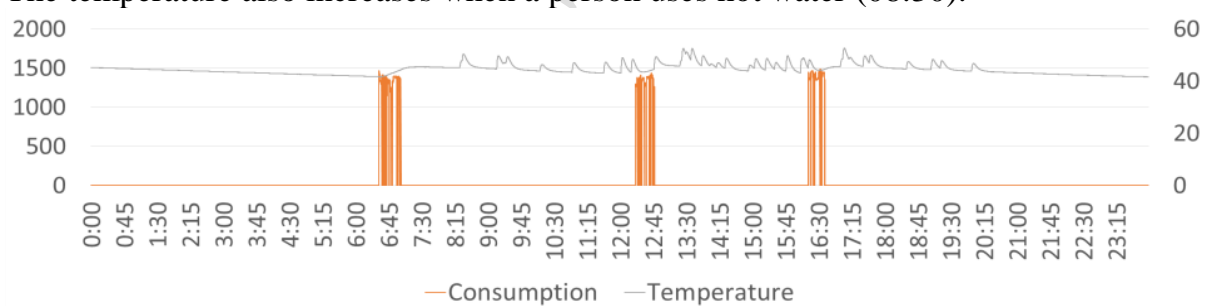

Fig. 5. Water Heater readings between 00:00 a.m. and 23:59 p.m.

\section{Conclusions}

The use of smart plugs can be included in demand side management systems. However, the information provided by the smart plugs, available on the market, are limited because they don't understand the load context, making it difficult to execute intelligent energy management algorithms.

The use of EnAPlug brings advantages for R\&D centers regarding load study and analysis. For power systems, EnAPlug has the advantage of environmental and contextual awareness that can be used for intelligent algorithms. EnAPlug is dynamic and easy to use in a R\&D center, enabling the sensing, monitoring and control of energy loads. It is a possibility, demonstrated in the present paper, to use EnAPlugs in a multi-agent system for power system simulation. 
The main contribution of this paper is the demonstration of a truly smart plug with environment awareness capabilities for energy management.

\section{References}

1. Dimeas, A. L., Hatziargyriou, N. D.: Operation of a Multiagent System for Microgrid Control. In: IEEE Transactions on Power Systems, vol. 20, pp. 1447-1455 (2005)

2. Kirschen, D.: Demand-side view of electricity markets. In: IEEE Transactions on Power Systems, vol. 18, no. 2, pp. 520-527 (2003)

3. Ye Yan, Yi Qian, Sharif, H., Tipper, D.: A Survey on Smart Grid Communication Infrastructures: Motivations, Requirements and Challenges. In: Communications Surveys \& Tutorials, IEEE, vol.15 no.1, pp.5-20 (2013)

4. Washom, B., Dilliot, J., Weil, D., Kleissl, J. Balac, N. Torre, N., Richter, C.: Ivory Tower of Power: Microgrid Implementation at the University of California, San Diego. In: IEEE Power and Energy Magazine, vol. 11, no. 4, pp. 28-32 (2013)

5. Stamp, J.: The SPIDERS project - Smart Power Infrastructure Demonstration for Energy Reliability and Security at US military facilities. In: IEEE PES Innovative Smart Grid Technologies (ISGT), Washington, DC, 2012, pp. 1-1 (2012)

6. Gomes, L., Silva, J., Faria, P., Vale, Z.: Microgrid demonstration gateway for players communication and load monitoring and management. In: Clemson University Power Systems Conference (PSC), Clemson, SC, 2016, pp. 1-6 (2016)

7. Faria, P., Vale, Z.: Demand response in electrical energy supply: An optimal real time pricing approach. In: Energy, vol. 36, no. 8, pp. 5374-5384 (2011)

8. Siano, P.: Demand response and smart grids - A survey. Renewable and Sustainable Energy Reviews, vol. 30, pp. 461-478 (2014)

9. Gomes, L., Faria, P., Fernandes, F., Vale, Z., Ramos, C.: Domestic consumption simulation and management using a continuous consumption management and optimization algorithm. In: IEEE PES T\&D Conference and Exposition, Chicago, IL, USA, pp. 1-5 (2014)

10. Tsui, K.M., Chan, S.C.: Demand Response Optimization for Smart Home Scheduling Under Real-Time Pricing. In: IEEE Transactions on Smart Grid, vol. 3, pp. 1812-1821 (2012)

11. Fernandes, F., Carreiro, A., Morais, H., Vale, Z., Gastaldello, D.S., Amaral, H.L.M., Souza, A.N.: Management of Heating, Ventilation and Air Conditioning system for SHIM plat-form. In: IEEE PES Innovative Smart Grid Technologies Latin America (ISGT LATAM), Montevideo, pp. 275-280 (2015)

12. Morais, H., Vale, Z., Pinto, T., Gomes, L., Fernandes, F., Oliveira, P., Ramos, C.: MultiAgent based Smart Grid management and simulation: Situation awareness and learning in a test bed with simulated and real installations and players. In: IEEE Power \& Energy Society General Meeting, Vancouver, BC, 2013, pp. 1-5 (2013)

13. Gomes, L., Fernandes, F., Faria, P., Silva, M., Vale, Z., Ramos, C.: Contextual and environmental awareness laboratory for energy consumption management. In: 2015 Clemson University Power Systems Conference (PSC), pp. 1-6 (2015)

14. Gomes, L., Lefrançois, M., Faria, P., Vale, Z.: Publishing real-time microgrid consumption data on the web of Linked Data. In: 2016 Clemson University Power Systems Conference (PSC), pp. 1-8 (2016)

15. Vinagre, E., Gomes, L., Vale, Z.: Electrical Energy Consumption Forecast Using External Facility Data. In: 2015 IEEE Symposium Series on Computational Intelligence, pp. 659664 (2015) 